\title{
Jean-Guy Vaillancourt
}

Sociologue, département de sociologie, Université de Montréal

(1981)

\section{"Deux nouveaux mouvements sociaux québécois: le mouvement pour la paix et le mouvement vert"}

\author{
Un document produit en version numérique par Jean-Marie Tremblay, bénévole, \\ professeur de sociologie au Cégep de Chicoutimi \\ Courriel: jmt sociologue@videotron.ca \\ Site web pédagogique : http://www.uqac.ca/jmt-sociologue/
}

Dans le cadre de la collection: "Les classiques des sciences sociales"

Site web: http://www.uqac.uquebec.ca/zone30/Classiques_des_sciences_sociales/index.html

Une collection développée en collaboration avec la Bibliothèque

Paul-Émile-Boulet de l'Université du Québec à Chicoutimi

Site web: http://bibliotheque.uqac.uquebec.ca/index.htm 
Cette édition électronique a été réalisée par Jean-Marie Tremblay, bénévole, professeur de sociologie au Cégep de Chicoutimi à partir de :

Jean-Guy Vaillancourt, "Deux nouveaux mouvements sociaux québécois: le mouvement pour la paix et le mouvement vert".

Un article publié dans l'ouvrage sous la direction de Gérard Daigle et Guy Rocher, Le Québec en jeu. Comprendre les grands défis. Chapitre 28 «Deux nouveaux mouvements sociaux québécois: le mouvement pour la paix et le mouvement vert » (pp. 791 à 807). Montréal: Les Presses de l'Université de Montréal, 1992, 812 pp.

Jean-Guy Vaillancourt est sociologue, au département de sociologie de l'Université de Montréal

[Autorisation formelle accordée, le 9 mars 2004, par M. Jean-Guy Vaillancourt de diffuser cet article.]

A1

Courriel : jean.guy.vaillancourt@umontreal.ca

Polices de caractères utilisée :

Pour le texte: Times, 12 points.

Pour les citations : Times 10 points.

Pour les notes de bas de page : Times, 10 points.

Édition électronique réalisée avec le traitement de textes Microsoft Word 2001 pour Macintosh.

Mise en page sur papier format

LETTRE (US letter), 8.5' x 11'”)

Édition numérique réalisée le 11 octobre 2004 à Chicoutimi, Ville de Saguenay, province de Québec, Canada.

C Fait avec

Macintosh 


\section{Table des matières}

Introduction

1. Le mouvement pour la paix

2. Le mouvement vert

Conclusion

$\underline{\text { Bibliographie }}$ 
Jean-Guy Vaillancourt,

“Deux nouveaux mouvements sociaux québécois: le mouvement pour la paix et le mouvement vert".

Un article publié dans l'ouvrage sous la direction de Gérard Daigle et Guy Rocher, Le Québec en jeu. Comprendre les grands défis. Chapitre 28 (pp. 791 à 807). Montréal: Les Presses de l'Université de Montréal, 1992, 812 pp.

\section{Introduction}

\section{$\underline{\text { Retour à la table des matières }}$}

Parmi les acteurs collectifs qui ont tenu une place centrale dans la mise en marche des changements considérables qui ont affecté la société québécoise durant les trois dernières décennies, il faut mentionner tout d'abord les partis politiques et les syndicats ouvriers, et aussi de nombreuses autres organisations économiques, professionnelles, médiatiques, éducatives, voire religieuses. Mais certains mouvements sociaux, tout particulièrement, ont joué un rôle crucial dans ces transformations économiques, sociales, politiques et culturelles. Le mouvement féministe, ainsi que les mouvements populaires et autogestionnaires sont deux de ces mouvements, mais il y en a d'autres. Certains sont plus anciens, comme le mouvement nationaliste, qui s'est radicalisé dès le début des années soixante avec la montée de l'indépendantisme, et le mouvement ouvrier, qui s'est institutionnalisé, non pas dans un parti politique, mais dans des organisations syndicales qui sont passablement politisées. Ces deux mouvements ont eu des hauts et des bas au cours des trente dernières années, mais ils demeurent importants, comme l'atteste par 
exemple la participation de plusieurs de leurs leaders à la Commission Bélanger-Campeau sur l'avenir constitutionnel du Québec.

Le mouvement coopératif est un autre mouvement québécois plus ancien, qui a laissé une forte empreinte sur la société québécoise actuelle. Ses racines remontent au début du siècle, et en moins de cent ans, il s'est ramifié en un réseau imposant de caisses populaires et de compagnies d'assurances, mais aussi de coopératives agricoles, d'habitation, de consommation et de production. Parmi les mouvements québécois plus récents qui ont eu une influence considérable durant la période 1960-1990, on pourrait aussi mentionner le mouvement des jeunes (surtout, mais pas exclusivement, étudiant), les mouvements urbains et régionalistes, le mouvement de protection des consommateurs, le mouvement de solidarité internationale et divers mouvements religieux, comme le mouvement charismatique.

Parmi tous ces mouvements sociaux qui se sont développés au Québec depuis le début de la Révolution tranquille, il y en a toutefois deux qui me semblent spécialement dignes d'intérêt et de mention: le mouvement pour le désarmement et la paix, et le mouvement de protection de l'environnement, plus communément appelés le mouvement pacifiste et le mouvement écologiste. Si je n'utilise pas d'emblée ces deux derniers termes, c'est qu'ils me semblent trop restrictifs, car les pacifistes et les écologistes ne représentent qu'une partie restreinte, c'est-à-dire l'aile la plus radicale, et parfois aussi la plus visible, de chacun de ces deux mouvements sociaux contemporains.

Le mouvement pour le désarmement et la paix et le mouvement de protection de l'environnement, que pour être bref j'appellerai ici le mouvement pour la paix et le mouvement vert, faute de meilleurs termes, sont souvent caractérisés comme étant de nouveaux mouvements sociaux, par opposition aux mouvements sociaux plus anciens comme le mouvement ouvrier et le mouvement nationaliste.

Selon certains sociologues européens, les nouveaux mouvements sociaux ont surgi après 1960, à partir des revendications inédites qui sont apparues à cause des conditions structurelles de type nouveau propres à cette période. Dans cette société industrielle avancée ou postindustrielle en émergence, de nouveaux types d'action collective ont surgi à cause de problèmes et de tensions issus d'une expansion économique rapide et des politiques redistributives de l'État-providence. Une série de crises se sont manifestées sur divers plans (emploi, environnement, course aux armements, qualité de vie, etc.), en même temps que la bureaucratisation et le contrôle technocratique croissants dans les diverses sphères de l'activité humaine et de la vie quotidienne. Les acteurs sociaux ont alors été conduits à essayer de reprendre le contrôle de leur identité en créant des réseaux communautaires basés sur la coopération et l'actualisation de soi, et en défiant les frontières établies de la 
politique institutionnelle traditionnelle. Selon une expression chère à certains théoriciens des nouveaux mouvements sociaux, ces mouvements tentent de se réapproprier des espaces de liberté dans la société civile en luttant contre l'appropriation étatique du tissu social.

Pour les théoriciens des nouveaux mouvements sociaux, il va sans dire que le mouvement vert et le mouvement pour la paix sont deux des plus intéressantes parmi ces nouvelles formes d'action collective. Un des problèmes avec certaines des théories des nouveaux mouvements sociaux, c'est que ces mouvements ne sont pas aussi nouveaux qu'on pourrait le croire à première vue. Des mouvements dits anciens partagent avec eux, à des degrés divers, certaines des caractéristiques qui sont censées être l'apanage exclusif des nouveaux mouvements sociaux. Le mouvement pour la paix et le mouvement vert ne sont pas nés ex nihilo avec les luttes étudiantes et la nouvelle gauche des années soixante, ils ne sont pas complètement différents d'autres mouvements antérieurs à cette période, et leur croissance ne s'explique pas uniquement par l'apparition de la société postindustrielle. Par ailleurs, ils sont vraiment nouveaux dans le sens qu'ils se développent de façon passablement originale et qu'ils luttent différemment pour faire face à de nouveaux problèmes et à de nouveaux enjeux. Ils articulent et réfléchissent des changements sociaux et idéologiques profonds apparus après 1960.

Les problèmes et les tensions quant à l'environnement et à la paix mondiale se sont manifestés différemment après 1960 et les enjeux ont changé passablement; les solutions proposées ont donc varié elles aussi. En somme, le mouvement vert et le mouvement pour la paix des trois dernières décennies continuent partiellement les mouvements semblables apparus antérieurement, même s'il y a eu une certaine rupture et beaucoup d'innovations durant les années dont nous traitons, étant donné les changements économiques, politiques et culturels considérables qui ont marqué cette période. 


\section{Le mouvement pour la paix}

$\underline{\text { Retour à la table des matières }}$

Pour ce qui est du mouvement québécois pour la paix, on pourrait faire remonter sa naissance assez loin, par exemple à l'opposition qui s'est développée au Québec à la participation canadienne à la guerre des Boers, ou encore à la lutte des Québécois contre la conscription au moment de chacun des conflits mondiaux.

Mais commençons plutôt notre examen avec la période qui suit 1945, c'est-à-dire après Hiroshima et Nagasaki, puisque les enjeux ont alors changé considérablement. Il n'est plus question alors de lutte contre la conscription, mais plutôt de lutte contre l'utilisation des armes atomiques et même contre les essais atomiques. Vers le milieu des années cinquante, on a vu se développer au Québec un tel mouvement contre la bombe atomique et aussi contre les essais nucléaires dans l'atmosphère. Toutefois, dès la signature du traité d'interdiction des essais atomiques atmosphériques, en 1963, il y a eu baisse des activités des groupes de paix un peu partout en Occident, y compris au Québec, où le mouvement avait quand même pris un certain temps à démarrer après la Seconde Guerre mondiale.

La guerre du Viêt-nam a redonné au mouvement pour la paix un nouveau souffle d partir du milieu des années soixante. On peut dire que dès ce moment-là, le mouvement pour la paix québécois, assez diversifié idéologiquement, a repris un certain essor, mais avec la fin de cette guerre, le mouvement se démobilisa de nouveau. Le plan de 1979 de l'OTAN en vue de moderniser les armes nucléaires déployées sur le sol européen, l'invasion de l'Afghanistan et l'arrivée au pouvoir de Ronald Reagan ont fait renaître un mouvement pour la paix assez différent des précédents. Cette troisième vague post-1945 a duré elle aussi un peu moins de dix ans. Au Québec, elle a atteint son apogée entre 1982 et 1986, comme ce fut le cas dans plusieurs autres pays occidentaux. Une centaine de groupes sont nés ou se sont alors ranimés, formant des coalitions et des alliances et organisant des manifestations et des actions qui ont eu un impact considérable sur l'opinion publique et même sur les dirigeants politiques. Plusieurs grandes campagnes, entre autres la Caravane de la pétition pour la paix, la campagne Un F18 pour la paix, la campagne La guerre n'est pas un jeu, la lutte contre les essais de missiles de 
croisières et celle contre les vols à basse altitude au Nitassinan, ont été menées durant les années quatre-vingt. Il y a aussi eu plusieurs grosses manifestations, entre autres les manifestations de Montréal, chaque année en octobre, qui permettaient d'évaluer la force du mouvement. Ce mouvement se distinguait du mouvement anti-bombe des années cinquante et soixante et du mouvement contre la guerre au Viêt-nam par des revendications plus nombreuses, plus variées et plus globalisantes. Sa préoccupation majeure était la course aux armements entre les deux superpuissances, et le danger du conflit nucléaire qui aurait pu en découler.

Parmi les nombreux clivages que l'on pouvait déceler à l'intérieur de ce mouvement des années quatre-vingt, celui entre non-alignés et alignés pro soviétiques a sans doute été le plus marquant, alors que les différences entre anglophones et francophones, entre hommes et femmes, entre non-violents et révolutionnaires, entre jeunes et vieux et entre les Montréalais et les militants des autres régions, sont restées quand même latentes. Les alignés pro soviétiques étaient alors assez peu nombreux, mais ils étaient bien organisés. Liés au Conseil mondial de la paix d'Helsinki et au Conseil canadien de la paix, ils animaient une revue, Zone libre, et deux bulletins de liaison, Québec-Monde du Conseil québécois de la paix, et Paix nouvelles de l'Alliance pour la paix du Québec.

Leur position était généralement celle du marxisme soviétique brejnévien, à savoir l'opposition à la guerre des étoiles, à la militarisation de l'Arctique, et à la collusion entre le Canada et les États-Unis. Leur stratégie préférée étant de racoller le mouvement syndical, la CEQ surtout, et d'essayer de contrôler l'ensemble du mouvement au Québec en recherchant la bénédiction des instances de paix pancanadiennes. Ils ont rarement réussi ce tour de force, mais ils ne se décourageaient pas lorsque les non-alignés les reléguaient à une place plus modeste.

Quant aux non-alignés, ils s'inspiraient plutôt du groupe européen pour le désarmement nucléaire END et des verts européens. Ils se dissociaient des deux superpuissances, et ils encourageaient l'émergence de mouvements pacifistes indépendants en URSS et en Europe de l'Est. Ils mettaient de l'avant des revendications très larges concernant le Tiers-Monde, la protection de l'environnement, et la défense des droits de la personne, en plus de lutter pour le désarmement et la reconversion industrielle, et contre le commerce des armes et les budgets militaires. Les non-alignés attiraient surtout les jeunes, les artistes, les étudiants, les écolos, les anarchistes ainsi que des progressistes libertaires, gandhiens, religieux ou socialistes.

La structure organisationnelle des groupes non alignés était très souple et se basait sur la démocratie de participation plutôt que sur l'autorité bureaucratique et hiérarchique. Ils prônaient l'action directe et non violente plutôt que 
les pressions sur les politiciens. De plus, les non-alignés faisaient spontanément le lien entre la lutte pour le désarmement et la paix d'une part, et les luttes féministes, écologistes, et tiers-mondistes d'autre part. Le Bulletin $d u$ Centre de ressources sur la non-violence, le groupe de paix québécois le plus dynamique (avec les Artistes pour la paix, qui est le groupe le plus nombreux), est la publication non alignée la plus intéressante à l'heure actuelle. Son analyse est généralement axée sur les conséquences écologiques, économiques, sociales et politiques néfastes de l'option militaire, et sur la nécessité de développer des moyens de défense non violents. La revue Option-Paix, par ailleurs, a représenté durant plusieurs années un éventail assez large de points de vues non alignés, mais à partir du numéro d'automne 199û, elle a pris une nouvelle orientation que d'aucuns qualifient de virage trotskiste.

Avec la fin de la guerre froide et surtout depuis l'irruption de la démocratie en Europe de l'Est, le mouvement de paix québécois, comme c'est le cas ailleurs, s'est retrouvé beaucoup moins souvent sur la brèche, mais il n'y a pas eu de démobilisation massive. Les groupes ayant profité des années de lutte plus intense, spécialement des appuis reçus durant l'Année internationale de la paix en 1986, pour s'institutionnaliser, ils ont pu continuer leur travail, même si les ressources ont diminué. Le mouvement ayant remporté certaines victoires, il n'est pas surprenant qu'un certain nombre de ses militants aient décidé de passer à autre chose à partir de 1988. Plusieurs des militants des groupes pour la paix se sont recyclés, c'est le cas de le dire, dans le nouveau mouvement vert qui a repris son essor au moment même où le mouvement pour la paix commençait à plafonner.

En effet, à partir de 1987 environ, les problèmes environnementaux globaux tels le réchauffement de l'atmosphère, les précipitations acides, la mort des océans, les déchets toxiques, la déforestation, la désertification et l'amincissement de la couche d'ozone, sont subitement apparus comme des menaces à la vie presque aussi dangereuses qu'une guerre nucléaire entre les superpuissances. En fait, de plus en plus de groupes perçoivent qu'il y a des liens étroits entre la crise de l'environnement, le mal-développement dans le Tiers-Monde, et la course aux armements, trois problèmes qui n'ont pas vraiment cessé de s'étendre dans plusieurs pays du Sud. Des groupes comme Greenpeace et Les Ami-e-s de la Terre, entre autres, qui relient les questions d'environnement, de développement et de désarmement, ont repris leur croissance à un rythme accéléré, après plusieurs années de relative accalmie. Toute une série de nouveaux groupes et de nouvelles coalitions font aussi leur apparition, et se situent d'emblée dans la ligne du développement viable (ou durable) préconisé par le rapport de la Commission Brundtland publié en 1987 sous le titre Notre avenir à tous. 
La guerre dans le Golfe arabo-persique, celle qui a fait suite à l'invasion du Koweit, a donné un regain de vie passager au mouvement anti-guerre québécois. Au début de 1991, il existait deux grandes coalitions contre cette guerre, I'une appelée Échec à la guerre, formée des groupes de paix plus anciens, tels les Artistes pour la paix, des trois grandes centrales syndicales et de diverses personnalités, dont l'écologiste Pierre Dansereau qui en a été le porte-parole à la conférence de presse du 23 janvier 1991, I'autre appelée Coalition contre la guerre dans le golfe arabo-persique, formée surtout de groupes d'étudiants des cégeps et des universités et de divers groupuscules de gauche, qui a pris des positions légèrement plus radicales et plus antiaméricaines que le premier regroupement.

Après la guerre du Golfe, des groupes comme les Artistes pour la paix, la Coalition pour l'abolition de l'armée, le groupe Nos Impôts pour la paix, le Centre des ressources sur la non-violence, l'Alliance pour l'action non violente, le Comité ZLAN de Montréal, le collectif Pacijou, la Coalition contre l'OTAN au Nitassinan, une nouvelle Coalition des femmes pour la paix, et divers groupes anglophones de Montréal entre autres, continuent leur action, en centrant l'attention sur le gaspillage des dépenses militaires, sur le commerce et la prolifération des armes, sur la conversion des industries et des budgets militaires, sur l'objection de conscience et sur la militarisation de la culture. À l'automne de 1991, une large coalition de groupes de paix, de syndicalistes, d'écologistes, de féministes, de tiers-mondistes s'est formée, sous le nom de Coalition québécoise pour la justice sociale et la paix, pour exiger entre autres une réduction de moitié du budget militaire canadien et le transfert des sommes ainsi économisées vers la conversion industrielle, la santé, l'éducation, l'environnement, le développement durable au plan international, les arts, les groupes de citoyens, etc. De plus, une grande enquête populaire sur la paix et la sécurité a fait une tournée du Québec pour écouter le point de vue des citoyens sur les réformes à entreprendre dans les politiques de défenses canadiennes, et la grande marche pour la paix du 27 octobre a porté sur le thème de l'art au service de la paix. C'est donc dire que le mouvement pour le désarmement et la paix reste bien vivant, même s'il n'arrive pas actuellement à mobiliser autant de gens qu'aux moments forts de la guerre froide. 


\section{Le mouvement vert}

$\underline{\text { Retour à la table des matières }}$

Le mouvement vert a véritablement démarré au Québec au début des années soixante-dix, même s'il y a des groupes conservationnistes plus anciens, tels les Jeunes naturalistes, qui font figure de précurseurs. À partir de 1970, de nombreux groupes environnementalistes se créent pour lutter contre la pollution et pour défendre l'environnement. Quelques années plus tard, certains groupes commencèrent à prendre une approche plus radicale, que nous appelons écologiste, et que certains caractérisent comme étant de l'écologie politique ou sociale, voire même profonde. J'ai souvent affirmé que tous ces groupes constituent une faune très diversifiée, dont il n'est pas facile d'établir un tableau précis, étant donné que les groupes sont de plus en plus nombreux et qu'ils évoluent rapidement. Le Répertoire environnemental du gouvernement du Québec en présente 706 dans son édition de 1990. Sur ce nombre, 544 (77\%) sont classés comme des groupes environnementaux proprement dits, et $162(23 \%)$ comme des groupes para-environnementaux. Les 544 groupes environnementaux sont divisés de la façon suivante dans ce répertoire:

\begin{tabular}{lrr}
\hline Groupe & Nombre & $\%$ \\
\hline Groupes de protection de l'environnement & 214 & $(39,3 \%)$ \\
Groupes de conservation des ressources & 22 & $(4,0 \%)$ \\
Groupes de recherche en environnement & 45 & $(8,3 \%)$ \\
Groupes d'éducation à l'environnement & 161 & $(29,6 \%)$ \\
Groupes de récupération et de recyclage & 45 & $(8,3 \%)$ \\
Groupes de loisir scientifique en environnement & 57 & $(10,5 \%)$ \\
\hline Total & 544 & $(100,0 \%)$ \\
\hline
\end{tabular}

Ces chiffres peuvent être trompeurs, car les groupes d'une même catégorie peuvent être très différents les uns des autres. Certains sont petits et oeuvrent à l'échelle locale, d'autres sont gigantesques et couvrent tout le Québec. Toutefois, tous peuvent être considérés comme faisant partie d'un vaste 
mouvement vert qui prend de l'importance de jour en jour. Ces groupes diffèrent non seulement quant à leur objet d'intérêt et quant à leur dimension, mais aussi par leur degré de radicalisme et selon que leur idéologie insiste davantage sur des solutions socio-politiques ou sur des solutions culturelles à la crise de l'environnement.

En ce qui concerne les orientations idéologiques, les groupes présentent donc des positions plus ou moins radicales et ce, tant sur le plan culturel que sur le plan. La typologie suivante présente les diverses idéologies que l'on peut rencontrer dans le mouvement vert québécois de la période 1970-1992.

Les trois orientations idéologiques du plan culturel sont celles qui visent l'amélioration de la qualité de l'environnement en questionnant les réalités culturelles de la société de consommation et de gaspillage. Elles proposent des actions et des modes de vie contre-culturels individualistes ou communautaires, ou bien alternatifs. Les personnes ou groupes agissant principalement sur ce plan favoriseront par exemple la santé holistique, l'agriculture biologique, l'alimentation naturelle, l'utilisation de produits de nettoyage biodégradables et les énergies douces, bref des pratiques en harmonie avec l'environnement. La revue Guide Ressources exprime bien les diverses tendances rencontrées chez ces verts contre-culturels, et parfois même chez les alternatifs.

Les actions axées davantage sur le plan socio-politique essaient de faire naître des changements structurels et socio-politiques par rapport à l'environnement. Les conservationnistes travaillent par exemple pour la protection d'un lac ou d'un boisé et pour l'utilisation efficace des ressources naturelles. Les environnementalistes interviennent pour que cesse la pollution sur une plus grande échelle, et ils exigent des gouvernements des actions appropriées. Les plus radicaux, c'est-à-dire les écologistes, pour leur part, critiquent le système de production industriel basé sur la dégradation de l'environnement et sur le gaspillage, et ils proposent une société alternative plus respectueuse de l'environnement. 
TABLEAU

Le mouvement vert québécois: orientations idéologiques

\begin{tabular}{|c|c|c|}
\hline $\begin{array}{l}\text { Les verts } \\
\text { les plus conservateurs }\end{array}$ & $\begin{array}{l}\text { Les verts modérés } \\
\text { ou réformistes }\end{array}$ & $\begin{array}{c}\text { Les verts } \\
\text { les plus radicaux }\end{array}$ \\
\hline \multicolumn{3}{|l|}{ Plan culturel } \\
\hline $\begin{array}{l}\text { 1. Les contre-culturels } \\
\text { individualistes } \\
\text { - approche thérapeutique } \\
\text { - nourriture saine } \\
\text { Plan socio-politique }\end{array}$ & $\begin{array}{l}\text { 2. Les contre-culturels } \\
\text { communautaires } \\
\text { - réseaux d'alimentation } \\
\text { naturelle } \\
\text { - coopérative autogérée }\end{array}$ & $\begin{array}{l}\text { 3. Les socioculturels } \\
\text { alternatifs } \\
\text { - culture alternative }\end{array}$ \\
\hline $\begin{array}{l}\text { 4. Les conservationnistes } \\
\text { - protection d'un lac } \\
\text { ou d'une espèce } \\
\text { menacée } \\
\text { - conservation de la } \\
\text { nature }\end{array}$ & $\begin{array}{l}\text { 5. Les environnementalistes } \\
\text { - lutte pour que cesse } \\
\text { la pollution } \\
\text { groupes de pression } \\
\text { urbains }\end{array}$ & $\begin{array}{l}\text { 6. Les écologistes } \\
\text { - projet de société } \\
\text { alternative }\end{array}$ \\
\hline
\end{tabular}

Notons que les six catégories de cette typologie ne sont pas étanches. Les actions des personnes et des groupes du mouvement vert peuvent être plus ou moins radicales, et donc varier non seulement dans le temps mais aussi selon les différentes positions sur l'axe socio-politique, même si l'idéologie ellemême reste fondamentalement conservationniste et conservatrice, comme c'est le cas par exemple de certains défenseurs de ce qu'on appelle l'écologie profonde. De même, il est possible que les stratégies d'action d'un groupe particulier soient orientées à la fois sur le plan culturel et sur le plan sociopolitique. Un groupe pourrait par exemple être engagé dans un réseau d'alimentation naturelle (contre-culturel communautaire) et mener des actions pour la protection d'un lac (conservationniste). Le Mouvement écologiste alternatif de l'UQAM par exemple, comme son nom l'indique, chevauche les deux catégories les plus radicales sur l'axe culturel et sur l'axe socio-politique: C'est le cas aussi du Monde à bicyclette qui essaie de changer les comportements et la législation, et qui est composé d'une grande diversité de verts. Une telle typologie demeure quand même utile pour la description d'un mouvement dans la mesure où l'on évite la catégorisation étanche lorsqu'on l'applique aux groupes et aux individus concrets. La diversité des orientations idéologiques et des actions nous oblige le plus souvent à analyser toute la dynamique pouvant exister entre les catégories et à reconnaître la complexité de la réalité par rapport aux modèles théoriques abstraits. 
Vers la fin des années soixante, à part quelques scientifiques et leurs sociétés savantes, quelques fonctionnaires et quelques groupes d'amants de la nature, il existait fort peu de personnes préoccupées par la protection de l'environnement au Québec. En 1970, STOP (Society to Overcome Pollution) puis la SVP (Société pour vaincre la pollution) apparurent, de même que le Conseil québécois de l'environnement, qui a longtemps publié le bulletin $D e$ toute urgence. En 1973, avec la crise du pétrole, le Comité pour la défense de la Baie James voit le jour. Dans les années qui suivent, le Mouvement pour l'agriculture biologique et puis le Monde à bicyclette sont formés. Vers 1975, le projet de construire une série de centrales nucléaires sur les bords du SaintLaurent fut mis en échec grâce en partie à l'opposition de nombreux groupes écologistes antinucléaires, tels le Regroupement pour la surveillance du nucléaire et les autres groupes qui formaient le Front commun anti-nucléaire, qui se transformera en 1978 en une coalition appelée l'Alliance Tournesol. Cette même année, à Québec, Michel Jurdant fonde Les Ami-e-s de la Terre. À la suite de la manifestation antinucléaire à Gentilly, environ 180 groupes verts se réunissent au lac Saint-Joseph près de Québec durant l'été 1978 pour former une large coalition verte et pour rédiger un manifeste écologiste. Dès l'année suivante, c'est la lutte pour obtenir un grand débat public sur l'énergie qui accapare une bonne partie des énergies du mouvement vert québécois.

Durant la première moitié des années quatre-vingt, plusieurs militants écologistes passent graduellement de la lutte contre le nucléaire civil à la lutte contre le nucléaire militaire. Mais à mesure que la guerre froide s'estompe et que les nouveaux problèmes environnementaux globaux prennent le devant de la scène (effet de serre, pluies acides, couche d'ozone, déforestation, etc.), le mouvement vert redémarre et reprend un second souffle après quelques années de stagnation au début de la décennie. Des groupes comme Greenpeace, la Fondation québécoise en environnement, l'Union québécoise de la conservation de la nature qui publie Franc-Nord, puis Franc-Vert, Environnement-Jeunesse (qui publie actuellement l'une des meilleures revues vertes, Enjeu), la FAPEL (Fédération des associations pour la protection de l'environnement des lacs), le Réseau des groupes écologistes du Québec, Les Ami-e-s de la Terre, la Fédération québécoise de la faune, l'Association québécoise de lutte contre la pollution atmosphérique, le Regroupement national des Conseils régionaux de l'environnement du Québec, entre autres, deviennent vers la fin de la décennie de grosses organisations, puissantes, avec des milliers de membres, des budgets imposants, des locaux, des permanents et des publications. À eux seuls, Greenpeace et la Fondation québécoise en environnement ont environ 100000 membres. Ces divers groupes ont une cote de popularité très élevée dans les sondages, et le degré d'expertise qu'on y rencontre est de plus en plus haut. Le mouvement vert devient en plus une force politique que les partis existants ne peuvent pas négliger. À mesure que la conscientisation écologiste augmente dans la population, la pression se fait plus forte pour que les problèmes d'énergie, d'agriculture, de foresterie, de 
pollution de l'air et de l'eau, de déchets soient résolus par des politiques et des actions qui tiennent compte de la fragilité accrue de l'écosphère. Les gouvernements d'Ottawa et de Québec disent accorder la priorité à l'environnement, si l'on en juge par les discours des politiciens et les plans qu'ils élaborent, mais lorsque vient le temps de passer à l'action, de voter les budgets, et de mettre en pratique les plans verts et les rapports d'enquête (par exemple le rapport Lacoste sur le BAPE et le rapport Charbonneau sur les déchets dangereux), l'on découvre rapidement que leurs vraies priorités sont tout autres. Même après les catastrophes de Saint-Basile et de Saint-Amable, et malgré la croissance des groupes verts dans presque toutes les villes et régions du Québec, et même dans plusieurs villages et quartiers, les gouvernements sont très lents à répondre aux demandes qui leur sont adressées. Il n'est donc pas surprenant que de plus en plus de citoyens se mobilisent dans des groupes $\mathrm{du}$ mouvement vert, et décident aussi de voter pour les partis verts, même si ceux-ci ont encore très peu de chances de faire élire leurs candidats aussi longtemps que le système électoral demeurera uninominal à un tour plutôt que proportionnel.

Une des caractéristiques actuelles du mouvement vert, c'est qu'il s'organise en diverses coalitions et réseaux qui s'intéressent aux différentes composantes importantes de l'environnement. La forêt est l'objet des préoccupations du Regroupement pour un Québec vert, l'énergie est le champ d'action d'HydroGlasnost, de la Coalition pour un débat public sur l'énergie, et du Comité Baie James, les pluies acides et la pollution de l'air sont surveillées par l'Association québécoise de lutte contre la pollution atmosphérique, les lacs et les rivières sont sous la garde de la SVP et de la FAPEL, les sols sont l'objet de préoccupations du Mouvement pour l'agriculture biologique, les déchets sont le champ d'action de la coalition de trente-cinq groupes mise sur pied par le groupe Action pour la défense de la nature (ADN) de Valleyfield, des GRIP (Groupes de recherche d'intérêt public), de la Coalition Action-Rebuts et du nouveau Front commun pour la gestion écologique des déchets, et les transports sont le champ d'action du Monde à bicyclette, de Transport 2000 et de Vélo-Québec. Quant à la faune et à la flore, spécialement les espèces menacées, elles sont l'objet de la sollicitude de très nombreux groupes. C'est le cas aussi pour l'éducation à l'environnement, pour la recherche en environnement et pour la protection du patrimoine.

Des organismes bien établis comme le Mouvement Desjardins, et même plusieurs organismes plus traditionnels encore, sentent le besoin de se mettre à l'heure de l'environnement et d'appuyer des initiatives de protection de l'environnement et de développement viable, ce qui incite plusieurs groupes verts à radicaliser leurs positions encore plus et à s'unir davantage pour pouvoir faire avancer les dossiers et pour ne pas être débordés sur leur gauche. Dans sa thèse de doctorat, l'écologiste-politicologue Gagnon (1989), parle de l'échec relatif des groupes écologistes parce que ceux-ci n'ont pas réussi à 
régler les mégaproblèmes environnementaux, et il a sans doute raison de juger assez sévèrement certains groupes verts, mais je pense qu'il est aussi exact de dire que le mouvement vert a eu beaucoup de succès, relativement parlant, à son actif. Tous les sondages montrent l'importance croissante de l'environnement dans toutes les couches de la société, et la force des groupes verts sur le plan idéologique et politique augmente continuellement. L'expertise écologique continue de croître, les perspectives se font plus globales et de plus en plus de gens passent des principes généraux et de la conscientisation éthique à des actions concrètes et à des comportements pratiques. La croissance du mouvement vert québécois, à l'heure où les Québécois sont à repenser leur appartenance au Canada, est un des signes les plus évidents de l'émergence d'une société civile plus active et plus indépendante de l'État. Au lieu de se laisser absorber par l'action politique partisane, les groupes verts, ceux de la tendance écologiste surtout, luttent pour maintenir leur autonomie, alors que les groupes conservationnistes et environnementalistes fonctionnent davantage comme des groupes de pression qui essaient d'influencer ceux qui prennent les décisions plutôt que comme un véritable mouvement social au sens tourainien du terme.

Les écologistes purs et durs et les alternatifs sont plus indépendants du pouvoir, plus autonomes et plus critiques à l'égard des gouvernements alors que les autres verts moins radicaux sont en train d'acquérir une plus grande influence sur le plan culturel et sur le plan politique, ce qui ne va pas sans une certaine intégration de leur part dans le système économique et dans la politique des partis. Dans son ensemble, le mouvement reste donc tiraillé entre le désir d'autonomie, qui le coupe du monde des décisions, et le désir de participer aux décisions politiques et économiques, avec tous les risques de récupération que comporte un tel choix.

\section{Conclusion}

$\underline{\text { Retour à la table des matières }}$

Les pages qui précèdent présentent et analysent brièvement deux mouvements sociaux québécois en pleine croissance depuis quelques années, le mouvement pour la paix et le mouvement vert. Ces mouvements sociaux ne sont pas des groupes, ni même des ensembles bien circonscrits de groupes, ni encore moins des «personnages » historiques, mais plutôt des objets créés par l'analyse, des espèces de vastes réseaux très fluides, de réseaux plus petits qui 
existent non seulement dans l'esprit des observateurs et des acteurs mais aussi dans la réalité socio-politique et culturelle.

Ces deux nouveaux mouvements sociaux ont des racines historiques assez anciennes mais ils sont surtout caractérisés par de nouvelles valeurs, de nouveaux buts, de nouvelles stratégies et des tactiques souvent tout aussi inédites. En plus de lutter avec de nouvelles formes d'action, ceux-ci s'expriment souvent à travers de nouvelles structures organisationnelles, souples et décentralisées, tout en faisant appel à des militants passablement différents, quant à leur origine sociale, de ceux qui formaient l'armature des anciens mouvements sociaux.

Sur le plan des valeurs, ce qui est mis de l'avant par ces nouveaux mouvements sociaux, c'est la qualité de la vie et des relations humaines, la sécurité commune, l'actualisation de soi, les biens non matériels, l'égalité entre tous les humains, la solidarité sociale, le respect de la nature et d'autrui, plutôt que la production de biens matériels et leur consommation effrénée, et la violence institutionnalisée.

Sur le plan des objectifs, les nouveaux mouvements sociaux tentent de se réapproprier le contrôle de leur autonomie à l'égard de l'État en vue d'établir une société plus juste, afin d'améliorer la situation de tous les groupes de la société. Leurs buts sont plus généraux (et aussi plus généreux) que ceux des anciens mouvements sociaux, qui cherchaient souvent à prendre le pouvoir politique pour une classe sociale ou pour un groupe national. Quant aux stratégies et aux tactiques de ces nouveaux mouvements sociaux, ce sont rarement celles de la politique traditionnelle, à savoir la lutte électorale et les pressions politiques, mais plutôt des moyens non conventionnels comme des manifestations, des teach-ins, des contestations bruyantes, des occupations de locaux, l'action extra-parlementaire, le travail éducatif, et la recherche de nouvelles solutions pratiques.

Pour ce qui est des nouvelles formes d'organisation qu'ils mettent de l'avant, elles sont basées sur la participation égalitaire, la décentralisation effective, la spontanéité, le consensus, les réseaux et les coalitions spéciales. Enfin, les participants de ces nouveaux mouvements sociaux ne sont pas originaires d'une seule classe sociale ni, comme nous l'avons vu, les défenseurs d'une seule position idéologique. Ce sont des gens venant de plusieurs horizons sociaux et politiques, même si ce sont souvent des gens qui sont tenus à l'écart des avantages de la société postindustrielle ou qui prennent position en faveur de ces derniers. Les jeunes, les femmes, les gens plus instruits, la nouvelle classe moyenne, les employés du secteur des communications, les membres des groupes ethniques et culturels dominés sont très présents dans ces mouvements. 
Offe (1984) a montré que les acteurs qui font partie de ces deux nouveaux mouvements sociaux que sont les mouvements pour la paix et pour la défense de l'environnement ne s'appuient pas, pour définir leur identité, sur des codes politiques (gauche-droite, libéral-conservateur) ni sur les codes socio-économiques qui correspondent partiellement à ces derniers (comme ceux de classe ouvrière ou classe moyenne, pauvre ou riche, rural ou urbain). Ils ordonnent plutôt l'univers du conflit politique selon des catégories qui peuvent s'appliquer au genre humain dans son ensemble, alors que d'autres mouvements sociaux anciens ou nouveaux font reposer leur représentation sur des caractéristiques qui excluent d'emblée certaines personnes. Dans ce sens, ces deux mouvements ont la possibilité d'intéresser beaucoup plus de monde, et par conséquent de permettre des solidarités beaucoup plus larges que la plupart des autres mouvements sociaux, qu'ils soient anciens ou nouveaux.

Des auteurs comme Touraine ou Lipietz vont même jusqu'à parler du mouvement d'écologie politique comme un lieu de convergence pour les autres mouvements sociaux alternatifs pour qui l'autonomie et la solidarité sont des éléments centraux et pour qui la résistance à la domination technocratique plutôt que la défense d'intérêts et les demandes à l'égard de l'État est crucial.

Hegedus (1989), pour sa part, pense que les mouvements sociaux des années quatre-vingt, à l'Est comme à l'Ouest, font appel à la conscience éthique en s'attaquant aux thèmes de la guerre et de la paix, de la faim et de la pauvreté, de la protection de l'environnement et des droits humains. Ainsi la société se crée elle-même grâce à des processus conflictuels qui élargissent la gamme des choix individuels et collectifs créateurs d'historicité. Pour elle, ce sont les mouvements pour la paix des années quatre-vingt, bien plus que le mouvement écologique antinucléaire, qui trace la voie pour la compréhension des problèmes de la société globale autocréatrice des années quatre-vingt-dix.

Quant à Habermas (1979), il affirme que ce sont les nouveaux groupes contestataires en général, de même que les mouvements religieux, qui peuvent jouer un rôle critique en période de transition.

Quoi qu'il en soit, depuis quelques années, le mouvement écologiste et le mouvement pour la paix ont de plus en plus tendance à se recouper, et à s'allier avec les mouvements de libération, de travailleurs, de femmes, de chrétiens politisés, de jeunes et de solidarité internationale. Cette convergence est facilement compréhensible, étant donné que ceux qui sont les plus préoccupés par l'avenir humain se rendent compte du fait que ceux qui dirigent économiquement et politiquement les pays les plus riches accaparent et gaspillent les fonds nécessaires au développement durable des pays pauvres, à la protection de l'environnement et à la satisfaction des besoins de base des plus démunis. 


\section{Bibliographie}

$\underline{\text { Retour à la table des matières }}$

AUCLAIR, S., J. ROYER et J.-G. VAILLANCOURT (1991), “Trois revues québécoises entre l'environnementalisme et l'écologisme" dans J. Pradès, J.-G. Vaillancourt et R. Tessier (dir.), Environnement et développement. Questions éthiques et problèmes socio-politiques, Montréal, Fidès, p. 273-293.

BABIN, R. (1984), L'option nucléaire. Développement et contestation de l'énergie nucléaire au Canada et au Québec, Montréal, Boréal Express.

BABIN, R. et J.-G. VAILLANCOURT (1984), "Le néo-pacifisme québécois", dans J.-G. Vaillancourt et R. Babin (dir.), Le mouvement pour le désarmement et la paix, numéro spécial de la Revue internationale d'action communautaire, vol. 12, no 52, automne, p. 2734.

BABIN, R. et J.-G. VAILLANCOURT (1987), "La régionalisation du mouvement pour la paix" dans B. Dumas et D. Winslow (dir.), Construction I destruction des idées. Alternances, récurrences, nouveautés, ACFAS, Les Cahiers scientifiques, $\mathrm{n}^{\circ}$ 53, p. 147-158.

BEAUDET, J.-F. (1990), L'autre révolution. Écologie et non-violence sur une planète en danger, Montréal, Fidès.

BÉLANGER, P.-R. et al. (1987), Animation et culture en mouvement. Fin ou début d'une époque? Québec, Presses de l'Université du Québec.

BERTELL, R. (1988), Sans danger immédiat ? L'avenir de l'humanité sur une planète radioactive, Montréal, La pleine lune.

BOOKCHIN, M. (1976), Pour une société écologique, Paris, Christian Bourgois.

BOSQUET, M. (1977), Écologie et Politique, Paris, Hachette.

BROWN, L. (1990), L'État de la planète 1990, Paris, EconomicaWorldwatch Institute.

CAPRA, F. et C. SPRETNAK (1984), Green Politics. The Global Promise, New York, E.P. Dutton Inc.

CARSON, R. (1962), Le printemps silencieux, Paris, Plon. 
COMMONER, B. (1973), L'encerclement, Paris, Seuil.

COMMONER, B. (1990), Making Peace with The Planet, New York, Pantheon Books.

COTNOIR, L. et J.-G. VAILLANCOURT (1988), "Les interventions du mouvement vert à l'aube du XXIe siècle" dans Environnement: aujourd'hui pour demain, Montréal, Association des biologistes du Québec, p. 202-211.

DANSEREAU, P. (1973), La terre des hommes et le paysage intérieur, Montréal, Leméac.

DUMESNIL, T. et P. DANSEREAU (1981), L'écologiste aux pieds nus, Montréal, Nouvelle Optique.

DUMONT, R. et G. BOILEAU (1990), La contrainte ou la mort. Lettre aux Québécois sur l'avenir de la planète, Montréal, Éditions du Méridien.

GAGNON, L. (1985), L'écologie. Le chaînon manquant de la politique, Préface de J.-G. Vaillancourt, Montréal, Les Éditions de l'Alternative.

GAGNON, L. (1989), L'échec macro-écologique, exigences des écologistes et outputs des systèmes politiques. Montréal, Université de Montréal (thèse de doctorat).

GOUVERNEMENT DU QUÉBEC (1990), Répertoire environnemental 1990, Québec, Les Publications du Québec.

HABERMAS, J.'(1979), Communication and The Evolution of Society, Boston, Beacon Press.

HEGEDUS, Z. (1989), "Social Movements and Social Change in SelfCreative Society", International Sociology, vol. 4, no 1, mars, p. 19-36.

JUQUIN, P. et al. (1990), Pour une alternative verte en Europe ? Paris, La Découverte.

JURDANT, M. (1976), Les insolences d'un écologiste, Sillery, Boréal Express.

JURDANT, M. (1984), Le défi écologiste, Montréal, Boréal.

LIPIETZ, A. (1989), Choisir l'audace, une alternative pour le XXIe siècle, Paris, La Découverte.

MAHEU, L. (1983), "Les mouvements de base et la lutte contre l'appropriation étatique du tissu social", Sociologie et Sociétés, vol. 15, no 1, avril, p. 77-92.

MELUCCI, A. (1983), "Mouvements sociaux, mouvements post-politiques", Revue internationale d'action communautaire, vol. 10, no 50, p. 13-30.

MILBRATH, L. (1984), Environmentalists: Vanguard of a New Society, Albany, State University of New York Press.

NASH, R. (1974), The American Conservation Movement, St. Charles, Forum.

NASH, R. (1990), American Environmentalism: Readings in Conservation History, New York, McGraw Hill.

OFFE, C. (1984), "Nouveaux mouvements sociaux et nouveaux mouvements pour la paix", dans J.-G. Vaillancourt et R. Babin (dir.), Le mouvement pour le désarmement et la paix, numéro spécial de la Revue internationale d'action communautaire, vol. 12, $\mathrm{n}^{\circ}$ 52, automne, p. 3539. 
PAEHLKE, R.C. (1989), Environmentalism and The Future of Progressive Politics, New Haven, Yale University Press.

PEPPER, D.M. (1984), The Roots of Modern Environmentalism, Dover, Croom Helm.

PRADES, J., J.-G. VAILLANCOURT et R. TESSIER (dir.) (1991), Environnement et développement: questions éthiques et problèmes socio-politiques, Montréal, Fidès.

QUÉBEC SCIENCE (1979), Face au nucléaire, Québec, Les Presses de l'Université du Québec.

ROGEL, J.-P. (1981), Un paradis de la pollution, Québec, Québec Science.

ROUSSOPOULOS, D. (1983), Our Generation Against Nuclear War, Montréal, Black Rose Books.

SHRAGGE, E., R. BABIN et J.-G. VAILLANCOURT (dir.) (1986), Roots of Peace: The Movement Against Militarism in Canada, Toronto, Between the Lines.

TOURAINE, A. (1978), La Voix et le Regard, Paris, Seuil.

TOURAINE, A. et al. (1980), La Prophétie antinucléaire, Paris, Seuil.

TOURAINE, A. (dir.) (1982), Mouvements sociaux aujourd'hui. Acteurs et analystes, Paris, Éditions ouvrières.

TOURAINE, A. (1984), Le retour de l'acteur, Paris, Fayard.

VAILLANCOURT, J.-G. (dir.) (1981), "Écologie sociale et mouvement écologique", numéro spécial de Sociologie et Sociétés, vol. 13, no 1, avril.

VAILLANCOURT, J.-G. (1982), Mouvement écologiste, énergie et environnement. Essais d'écosociologie, Montréal, Éditions Albert SaintMartin.

VAILLANCOURT, J.-G. (1985a), "Le mouvement vert québécois: entre l'écologie et l'écologisme", Possibles, vol. 9, no 3, p. 3546.

VAILLANCOURT, J.-G. (1985b), "Les verts du côté de la colombe", Autogestions, vol. 20, no 21, p. 35-39.

VAILLANCOURT, J.-G. (1987a), "Écologie et néo-pacifisme”, dans P. Bélanger et al. (dir. ), Animation et culture en mouvement. Fin ou début d'une époque, Québec, Presses de l'Université du Québec, p. 171-178.

VAILLANCOURT, J.-G. (1987b), "Défense de l'environnement: les mouvements québécois", Relations, no 536, décembre, p. 298-301.

VAILLANCOURT, J.-G. (1987c), "L'enracinement du mouvement pour la paix", dans P. Laplante et J. Lévy (dir.), La paix, nouvelles avenues, Montréal, Éditions du Méridien, p. 167-183.

VAILLANCOURT, J.-G. (1988), "Le mouvement de paix face à la neutralité canadienne", dans C. Bergeron et al. (dir.), Les choix géopolitiques du Canada. L'enjeu de la neutralité, Montréal, Éditions du Méridien, p. 243-264.

VAILLANCOURT, J.-G. (1990), "Désarmer pour développer autrement: les mouvements pour la paix et contre le gaspillage des dépenses militaire", dans $\mathrm{R}$. Babin et S. Leblanc-Rainville (dir.), La paix comme projet de justice, ACFAS, Les Cahiers scientifiques, no 74, Montréal, p. 47-69. 
VAILLANCOURT, J.-G. et B. PERRON (1992), "Environnement et développement durable dans les mémoires collectifs soumis à la Commission Bélanger-Campeau", dans J. Pradès, R. Tessier et J.-G. Vaillancourt (dir.), Gestion de l'environnement, éthique et société, Montréal, Fidès.

WORSTER, D. (dir.) (1973), American Environmentalism: The Formative Period, 1860-1915, New York, Wiley.

ZALD, M.N. et J.M. McCARTHY (dir.) (1987), Social Movements in An Organizational Society, New Brunswick, Transaction Books.

Jean-Guy Vaillancourt,

Département de Sociologie, Université de Montréal

Fin du texte 